\title{
Las derivaciones de la identidad absoluta
}

\author{
The derivations of absolute identity
}

\author{
RODRIgo LAERA \\ Universidad de Barcelona \\ rlaera@uade.edu.ar
}

RESUMEN

El presente trabajo tiene como fin discernir entre tres tipos de identidad en el marco de la ontología formal: la absoluta, la simple y la identitaria. A partir de estas distinciones se propone la subsistencia de un panteísmo de la identidad que es entendido como un simple presentarse sin nada que se presente.

Palabras claves: identidad, ontología formal, diferencia, presentación

\section{Abstract}

This paper aims to distinguish between three types of identity within the framework of formal ontology: absolute, simple and identitary. As a result of aid differences, the subsistence of a pantheism of identity is upheld as simply presenting itself with nothing being presented.

Keywords: identity, formal ontology, difference, presentation.

\section{Introducción}

El problema ontológico de la identidad puede resumirse en dos preguntas generales: (a) ¿cómo algo, que es igual a sí mismo, pasa a ser algo distinto de sí? (b) ¿Cómo lo idéntico en sí puede desplegarse en múltiples determinaciones? Considerando que estas preguntas tengan algún sentido, debemos anteponer algunas observaciones generales respecto al propósito de la cuestión misma.

En primer lugar, importa distinguir el aspecto personal del aspecto físico de estas cuestiones. Es distinto pensar que un sujeto $S$ es idéntico en el tiempo a sí mismo, de considerar que un objeto físico es idéntico a sí mismo en 
el tiempo. ${ }^{1}$ En este trabajo no se considerarán ninguna de las dos posiciones. En segundo lugar, hay dos aspectos lógicos que consideran que la identidad es un principio constitutivo. Por un lado, se encuentra el análisis lógico-proposicional, que hace de la identidad una relación entre elementos equivalentes. Por otro lado, se encuentra el proyecto lógico hegeliano, y que el propio Hegel ha llamado esencialidades. Este proyecto no sólo abarca las categorías de identidad, diferencia y contradicción, sino también la esencia de la cosa pensada.

Dejando de lado ambos análisis de la identidad, este trabajo busca indagar en las derivaciones de una identidad ontológicamente absoluta. La importancia de este tipo de examen no es arbitraria, sino que responde a un vacío analítico en torno a la tematización ontológica que se conduce bajo criterios de identidad. En el siguiente apartado se establece una diferencia entre la identidad ontológica y el principio identitario de sustitución de los indiscernibles. En el tercer apartado se busca definir -si esto es posible- el carácter ontológico de la identidad absoluta, a partir del cual se comprenden las distintas identidades derivadas; en el cuarto apartado, la identidad.

\section{El principio de identidad y de sustitución de los indiscernibles}

Uno de los principios fundamentales que rigen la identidad es el principio leibniziano de indiscernibilidad de los idénticos. ${ }^{2}$ Bien entendido, este principio no debe confundirse, como en ocasiones se hace, con el principio de sustitución. ${ }^{3}$ El primero se refiere a la captación de una relación que es en sí misma inmediata, mientras que el segundo indica que cada miembro de una identidad puede ser reemplazado por el otro en cualquier contexto, que no por ello alterará la verdad del resultado. Parece relativamente sencillo darse cuenta que el último principio es más complejo que el primero, ya que no solamente expresa un algo puesto en tanto unidad que se relaciona, sino que también implica la noción de un "contexto"; es decir, de un conjunto que no es ese "algo puesto". ${ }^{4}$ De esta manera, se presupone que si dos objetos "a"

1 Donde, si $\mathrm{Y}$ es idéntica a $\mathrm{X}$, entonces necesariamente, $\mathrm{X}$ e $\mathrm{Y}$ llenan la misma región del espacio-tiempo.

2 Una manera de enunciar el principio de indiscernibilidad de los idénticos puede ser la siguiente: si, para cada propiedad $F$, un objeto $x$ posee $F$ si y sólo si el objeto $Y$ tiene $F$, entonces $x$ es idéntico a $Y$.

3 Quine, W. V., Desde un punto de vista lógico, Madrid: Orbis, 1984.

4 Cuyo modelo lingüístico es el intercambio sinónimo por sinónimo, que tanto ha criticado el propio Quine. Compárese también con TYE, M., "A note on the synonymy principle of proper- 
$\mathrm{y}$ " $\mathrm{b}$ " son idénticos, entonces, si " $\mathrm{a}$ " tiene cierta propiedad " $\psi$ ", también la tiene " $\mathrm{b}$ ", admitiendo que esta propiedad tiene el mismo valor de verdad en cualquier contexto asignado a "a" como en cualquier contexto asignado a " $b$ ". En ambos casos, se concibe la identidad como una relación psicológica: una cosa que no somos capaces de distinguir respecto de otra relacionada equivale a que algo es idéntico a algo.

Por un lado, se podría agregar que hay una necesidad de avanzar teoréticamente sobre identidades, estableciendo designadores rígidos para poder ampliar el principio de sustitución. ${ }^{5}$ Así, el siguiente paso sería llegar a sostener que no hay mundos posibles que sean distintitos y que sean cualitativamente indiscernibles. ${ }^{6}$ Por otro lado, también se puede pensar que un objeto que carece de distinción de sí, en el tiempo-sucesión-, equivale a que es idéntico consigo mismo - unidad. Aunque las dos equivalencias ya presuponen cierto principio de identidad, la primera aspira a la relación en sí misma, mientras que la segunda, a la unicidad del objeto en el tiempo. Visto de ambas maneras, el análisis lógico-psicológico del principio de identidad parece tener poco que ver con el examen metafísico de la identidad, aunque en muchos casos ambos puedan ser concebidos como semejantes. La semejanza más notoria es que, tanto el principio de indiscernibilidad como el de sustitución, están condicionados por algo externo de suyo: el principio de indiscernibilidad necesita de algo que discierna y que, siendo el caso, no pueda ser discernido; el principio de sustitubilidad necesita de algo que sustituya y de algo que, siendo el caso, no se pueda sustituir. En los dos principios hay cierta dependencia respecto del estado psicológico de quien discierne o sustituye, pues no se dice que, al ser " $x$ " igual a sí misma, no se puede distinguir, sino que, al no poder distinguirse, " $\mathrm{x}$ " es igual a sí misma.

$\mathrm{Al}$ estar centrados en la diferencia cognitiva, los dos principios chocan con una dificultad de fondo: si la identidad depende de nuestra capacidad de discernir, uno puede preguntarse cuántas veces hay que no distinguir una cosa de otra para saber que son indistinguibles. Teniendo como resultado una proyección infinita de veces, nunca sabremos a ciencia cierta si algo es idéntico o no. Por lo tanto, algo es idéntico hasta que deja de serlo; de manera que la identidad es un presupuesto, una hipótesis o, en el peor de los casos, algo tan frágil como un prejuicio, encontrándonos ante el callejón sin salida de un relativismo meta-epistemológico.

ty identity. Analysis", vol. 42, núm. 1, 1982, pp. 52-55; para quien la propiedad $p$ es idéntica a la propiedad $q$ si y sólo si cualquier predicado $F$ y $G$, si $p$ es expresable por $F$ y $q$ es expresable por $G$, entonces $F$ es sinónimo de $G$.

5 Cf., LaPorte, J., Rigid designation and theoretical identities, Oxford: Oxford University Press, 2013.

6 Para confrontar esta idea, Cf. Lewis, D., On the Plurality of Worlds, Oxford: Blackwell, 1986. 
La mera noción de distinción no basta para que la identidad sea tomada como un principio dependiente de la psicología. El que dos cosas conformen una identidad es un hecho que puede partir, o bien de la propia identidad, o bien de nuestra incapacidad de distinguirlas o, en el último de los casos, de nuestra capacidad o incapacidad psicológica de relacionarlas o de componerlas como una unidad, conformando un criterio. ${ }^{7}$ Únicamente en el segundo y tercer caso diremos que la identidad es dependiente de la psicología. Por un lado, la distinción se revela como muestra de una asimilación; pero, por otro lado, la distinción encuentra en la determinación su diferencia más conveniente, siendo ajena, en sí misma, a nuestra capacidad de relación y comparación.

Aun cuando la identidad en sí misma no implique un acto de comparación, debe haber un algo exterior que relacione identidad con indiscernibilidad o indiscernibilidad con identidad, por lo cual la identidad que puede llegar a ser captada no es nunca una identidad absoluta u original, sino una identidad derivada, cerrada, que exige algo que se eleve sobre ella para establecerse como tal. En los dos casos queda expuesta una psicología de la identidad, en donde (1) el acontecimiento psíquico que unifica se eleva sobre una posible formación conjunta siendo ya mediación, es decir, razón de una unidad de la multiplicidad; (2) el acto unitario de aprehensión que divide, establece por medio de la analogía una dualidad identitaria, es decir, una multiplicidad simétrica de la unidad. Ahora bien, resta preguntarnos: ¿en qué medida es posible trascender los principios psicológicos de la identidad?

Está claro que, si apostamos por semejante trascendencia, debemos elevarnos sobre ambos principios. Ya que, si bien es cierto que uno es más simple que el otro, ninguno de los dos nos permite captar la esencia de la identidad, ni nos es de gran ayuda para conseguir una respuesta formal a las dos preguntas con las que abrimos este escrito. Aun así, debemos dar cuenta de nuestras limitaciones: toda exposición acerca de la identidad está, desde luego, mediada por el concepto. Esta mediación es inevitable, y únicamente puede ser superada por medio de una abstracción que implique el vaciamiento de contenido efectivo que se encuentra determinado por el signo mismo. Podría pensarse que el signo envuelve, de alguna manera, un contenido, ya que (necesariamente) el signo es signo de algo. Sin embargo, el concepto que confinado al signo es dador de sentido, puede tornarse meramente abstracto e indeterminado. Si el concepto, que es en sí mismo determinación, se vuelve en lo esencial indeterminado, entonces el signo queda liberado de referencia

7 Lowe, E., "What is a criterion of identity?", en Philosophical Quarterly, vol. 39, núm. 154, 1-29; HaWley, K., "Principles of composition and criteria of identity", en Australasian Journal of Philosophy, vol. 84, núm. 4, 2006, pp. 481-493. 
y, más aún, de sentido. El concepto indeterminado es un concepto anulado, es decir, vaciado, y el signo, al tener como correlato un concepto vacío, se transforma en mera marca. Revertirse en una mera marca significa que el signo carece de contenido y en consecuencia no expresa nada. A raíz de esto, el concepto, ahora alejado de la propiedad del signo, se vuelve externo a él quedando relegado a un segundo plano, digamos, explicativo de la mera marca. El concepto queda dispuesto como "lo externo", es decir, como suplemento de la marca para conferirle efectividad, y pasa a ser, simplemente, mediación respecto a su ausencia.

Sintetizando, en el principio de indiscernibilidad y en el de sustitubilidad, lo principal era el acto psíquico para la formación del concepto, la confusión surgía porque aquello que es la consecuencia de la identidad era entendido como la causa, a la vez que tanto la causa como la consecuencia eran tomadas como necesarias, sin distinguir entre lo que sería "conciencia de identidad" e "identidad en sí misma". Esta confusión lleva consigo un prejuicio elemental: el de creer que todos los principios deben ser consecuencia de representaciones de hechos. Este prejuicio, unido a tal confusión, no nos permite ver con claridad la diferencia entre el enunciado y aquello a lo que el enunciado refiere. Lo psíquico, en este caso, estaría en la realización del enunciado de identidad, por ejemplo " $a=a$ ", y no en la identidad en sí misma -que se encuentra representada por " $a=a$ ". Ahora bien, si la aprehensión de la identidad únicamente puede llevarse a cabo especulativamente mediante el concepto, y si el concepto es por definición "determinación", nuestro análisis está sujeto a determinaciones que son, para el caso, ineludibles. La aprehensión entonces es "aprehensión especulativa", y como tal no puede captar plenamente el objeto sino tan sólo aproximarse a él. Que la aprehensión no sea plena sino aproximada, quiere decir ni más ni menos que no aprehendemos el hueso sino la cáscara. Sin embargo, esta aproximación es característica de la forma lógica que reviste la identidad absoluta. Viviendo como vivimos en la determinación de las cosas, es necesariamente imposible por definición situarnos en la identidad absoluta, solamente es posible exponerla objetivándola, siendo "lo otro" que ella es y, por lo tanto, al ser "lo otro", haciendo que ella deje de ser absoluta. A pesar de esta imposibilidad última, nada nos impide referirnos a ella en tanto eidos.

El análisis ontológico formal debe aspirar a captar aquel saber que produce el objeto, la idea, que teniendo como órgano el enlace entre pensamiento y objeto debe superar el conocimiento inferencial: ni puede entenderse como una creencia que es adquirida como resultado de mantener otras creencias, ni como el estado en el cual una creencia es sostenida sobre la base de otras. Por el contrario, el análisis ontológico formal de la identidad reclama un fundamento intuitivo, que a la vez sea producto del pensamiento especulativo. 
He aquí la doble cara de dicha aproximación. De un lado es mediación por parte del pensamiento especulativo con el cual se constituye como una idea, del otro es inmediación en cuanto fundamento intuitivo, un fundamento que se brinda originariamente por sí mismo y que confiere unidad teórica a sus posibles derivaciones: una constitución ontológica de la identidad.

\section{La identidad absoluta como mera marca}

Pero aún queremos ser más justos, y para eso se debe especificar qué concebimos como "identidad absoluta", para luego estar en condiciones de desarrollar sus determinaciones formales. Por "identidad absoluta" entendemos aquel "algo" cuyo contenido no es más que su propia manifestación. A razón de esto, diremos que la identidad absoluta es un mero marcar, vacío, completamente abstracto, desprovisto de forma y contenido. ${ }^{8}$

En cuanto intuición original y producto de una reducción del pensamiento especulativo, la identidad absoluta viene determinada como unidad-totalidad ideal (Ser ideal); empero, ella es propiamente indeterminada, de modo que no puede ser en sí misma eidos, sino algo todavía más elemental y abstracto, pues el eido, , en cuanto "forma", asume cierta especificidad que es extraña a la simple manifestación. En este sentido, la identidad absoluta puede concebirse negativamente como una ausencia, a la vez que positivamente como el "presentarse" de esa ausencia: la manifestación de la ausencia. Dicho "presentarse", como pura manifestación, carece de contenido. De hecho, al asentarse mediante ideación en un mero marcar informe, la manifestación no puede ser manifestación de algo más que sí misma: manifestación de manifestación y así al infinito. Es importante, por lo tanto, tener en cuenta que si el origen de la identidad, la identidad absoluta, es como dijimos "mero marcar", entonces no puede ser presentada como primer principio bajo la forma " $a=a$ " o, peor aún, bajo la forma " $a$ es $a$ ", puesto que en la forma " $a=a$ " ya impera un desdoblamiento, que parte desde un primer " $a$ " ya "puesto" (la marca en sí misma), culminando en un segundo " $a$ " que podría ser tomado como su imagen (marca de marca), si lo leemos linealmente de derecha a izquierda. Así, la universalidad de la identidad absoluta consiste en su singularidad, en ser ella misma "manifestación absoluta", en sí misma inseparable e

8 Geach sostiene que la noción de absoluta identidad no tiene aplicación, concluyendo que la identidad solamente puede ser una relación. Siguiendo esta ya clásica línea, aunque quizás de manera más radical. GEACH, P., "Ontological relativity and relative identity", en Munitz, M. K., Logic and Ontology, New York: New York University Press. Cf. también: Griffin, N., Relative Identity. Oxford: Oxford University Pressm 1977. 
indeterminada. Al haber en " $\mathrm{a}=\mathrm{a}$ " una distinción, una separación intrínseca, aunque sea sencillamente formal, no puede representar la identidad absoluta sino algo que es derivado de ella. Retomaremos este punto más adelante.

Si la identidad absoluta se muestra necesaria por obra del concepto, entonces la reducción eidética y el pensamiento deben estar unidos, por lo que lo psíquico estaría en la realización del enunciado y no en lo que se enuncia, en sí mismo, alcanzando la distinción entre el aparecer puro y el "cómo" del aparecer. Pues, siendo que la unidad del concepto es lo efectivo, la universalidad de la identidad absoluta consiste en su singularidad, que es en sí misma inseparable e indistinguible. La identidad absoluta, al ser concebida como principio intelectivo fundamental, no puede tratar de la unidad del pensamiento y del ser como sucede por ejemplo en Descartes, sino de la indistinción de pensamiento y Ser. El contraste estaría en que uno plantea la fórmula "pensamiento $=$ Ser", mientras que una indistinción entre ambas categorías superaría la propia equivalencia, ya no siendo un reflejo de sí, sino la ausencia de todo reflejo o toda reflexión: el marcar sin marca. Por consiguiente, la identidad absoluta no es algo "puesto" como sería un simple " $\mathrm{A}$ ", porque de ser así habría algo que "pone" y su contenido sería algo más que propia manifestación, sería la manifestación de "algo otro".

Así, la identidad absoluta es (1) simplemente manifestación que se manifiesta, así hasta el infinito. Al ser solamente manifestación, carece de propiedades, por lo cual es en sí misma (2) vacía y puramente abstracta. Si su contenido es simplemente manifestación, entonces (3) debe consistir en un aparecer puro, superación de todo reflejo o toda reflexión; por lo que no puede ser un producto del pensamiento, sino algo independiente de él. Aquello que tiene su manifestación como su propio contenido se corresponde con un (4) mero "marcar". Y como mero "marcar", no puede consistir en un desdoblamiento ni en una reproducción de sí misma como lo sería la ecuación "a = a". Al ser mero "marcar" es también (5) "forma" pura. Más precisamente, es la indistinción de forma y contenido: siendo el contenido la forma y la forma el contenido, es decir su propia manifestación.

El origen, el fundamento, llega a ser una marca privada de concepto, fijada por el vaciamiento del concepto: lo pensante abstracto. De aquí parte toda determinación, de un marcar vacío que es "constitución originaria", es decir, "constitución no constitutiva". La identidad absoluta es una identidad originaria, pues no hay pliegue, no hay recursividad y al ser completamente vacía de contenido no puede estar "puesta", por lo que debe distinguirse también de la identidad simple que supone "A".

Tampoco podemos decir que esta identidad absoluta sea forma de todas las formas, ni contenido de todos los contenidos, ni marca de todas las mar- 
cas, en el sentido que están "siempre ya" perteneciendo unas a otras. Porque con esto afirmaríamos algo más que su propia manifestación, que su desolada insistencia. No obstante, podemos apuntar que es posibilidad de marcas, de formas, de contenidos. Es más, podemos decir que su absoluta posibilidad es su absoluta necesidad, y viceversa. En este sentido, la identidad absoluta como pura manifestación es (6) pura posibilidad. Siendo la posibilidad una abstracción vacía, indistinta e indeterminada, el mero marcar por estar libre de contenido puede entenderse como libertad absoluta. Pero a la vez, siendo manifestación de manifestación de sí misma hasta el infinito, es lo que siempre ya subsiste: su propia necesidad. Por lo tanto, la identidad absoluta se entiende como un simple presentarse sin nada que se presente, un marcar en el que yace también la ausencia por ser vacío en contenido.

No está de más advertir que en la identidad absoluta no se halla nada que pueda pensarse como "latente", es decir, algo que está una vez presente, otra ausente, porque eso indica que hay un estar previo. ${ }^{9}$ Por el contrario, tampoco uno debería estar tentado a pensar que se trata de un lugar de convergencia; en el vacío del mero marcar se halla el desarrollo de un algo independiente -en principio- de toda convergencia con arreglo a lo cual la posibilidad es inmediata a la necesidad de la manifestación de la manifestación, etc. Puesto que la identidad absoluta no soporta una estructura representativa, ya sea por medio de una relación, ya por medio de una auto-referencialidad, debe ser, completamente, actividad abstracta de sí misma. En consecuencia, como mero marcar que repite su mero marcar, es ajena a las consideraciones fijas del entendimiento. Este "fijar" del entendimiento es más acorde [a] la identidad simple.

\section{La diferencia entre identidad absoluta fundamental e identidad simple como primera forma}

La identidad absoluta no es el único tipo de identidad que encontramos en una ontología formal. De ella brotan otras formas de identidad que, por estar subordinadas en origen, las llamamos "identidades derivadas". Hemos dicho en el parágrafo anterior que la identidad absoluta encierra la pura posibilidad (en el sentido que es posibilidad pura de la necesidad y necesidad de

${ }_{9}$ Quizás sea imprudente no dejar claro lo siguiente: cuando decimos, en el caso de la identidad absoluta, que su manifestación es ausencia y su ausencia manifestación, y que su necesidad es posibilidad y su posibilidad es necesidad, estamos intentando dar un perfil de aquello que se capta como indistinto por medio del pensamiento y que tiene ser expresado de alguna manera. Es evidente que si aspiramos a un solemne rigor lo mejor sería condenar la descripción de esta identidad al silencio, pero la consecuencia sería renunciar al fundamento, que es, en definitiva, lo único importante, desde luego infinitamente más importante que el rigor profesional. 
la posibilidad). Esta posibilidad, como libertad incondicionada y actividad sin límites, no alcanza a manifestarse como mera marca, a constituirse como realización. La manifestación de la manifestación... etc. se constituye por necesidad como lo realizado: actividad realizada. Con lo cual deviene en un "algo puesto", puesto en sí mismo, una auto-posición que podríamos llamar "identidad simple": "A". Hemos pensado a la identidad absoluta como lo ilimitado (indiferencia absoluta), como el impulso infinito de la pura presencia que era mera marca. Mediante su posibilidad inmanente, la identidad absoluta deviene en sí misma, trascendiéndose a "lo puesto". La trascendencia de sí, propia de su actividad (manifestación de manifestación...etc.), culmina en lo "puesto", en la presentación de la manifestación de la manifestación... etc. Claro está que "lo puesto", la presentación, en este caso no puede ser dado "desde afuera", sino que es producto de la propia actividad, en este sentido decimos que se trata de una (auto) presentación. ${ }^{10}$

Concibiendo la identidad simple como el desarrollo unificado de la totalidad absoluta en tanto posibilidad de presentación, la cohesión de lo singular y lo plural ("uno-todo" abstracto) empieza a cobrar forma, no ya como mero marcar, sino como mera marca. Decimos, entonces, que la identidad simple es ya formal, en donde lo "puesto de sí" es la presentación. Si la identidad absoluta era un marcar vacío e indeterminado, libre de la dualidad forma-contenido, en la identidad simple esta dualidad empieza a ser efectiva por haber un principio de forma, aunque su contenido sea todavía abstracto. Con lo cual, la (auto) presentación debe distinguirse de la "propia manifestación" de la identidad absoluta, ya que el "ser puesto" de la identidad simple, su inmediatez, es superado por la actividad de su propio aparecer. Para decirlo de una manera sencilla, mientras que la (auto) presentación de la identidad simple es la forma de un aparecer, la identidad absoluta es absolutamente informal: la una se auto-presenta vacía, pero con un principio de forma; la otra se manifiesta sin ningún tipo de principio, ya que el fundamento es ese manifestarse vacío.

Recién en la identidad simple encontramos un umbral de síntesis, en tanto que opera como "unidad", "unidad formal". Sin embargo, todavía podemos preguntarnos cómo es que en la identidad simple están estos principios que en la identidad absoluta faltan, siendo que la una es derivada de la otra.

La realidad de la manifestación, vacía, de la identidad absoluta es la actividad de la posibilidad efectiva. Posibilidad que se encuentra, en este caso, con la posibilidad de "lo otro" como condición de lo mismo que, en cuanto es posible, es desde luego contingente. La identidad simple es una contingencia

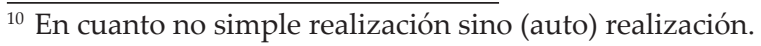


de la necesidad que supone la indeterminación de la identidad absoluta como manifestación originaria. Que la identidad absoluta sea anterior a la identidad simple, ya que esta última es efectividad de la posibilidad que encierra a la primera, no significa que aquí entre en juego la temporalidad. El problema del tiempo aún no ha surgido, por lo tanto, la anterioridad a la cual nos referimos en una anterioridad estrictamente formal. De este modo, la posibilidad pura de la manifestación de la manifestación... etc., que se considera como identidad absoluta, se hace efectiva en un "poner" formal, una mera marca. Esta marca, siendo lo formalmente "puesta", conforma la identidad simple: "A".

No obstante, ha quedado la siguiente pregunta en el camino: ¿cómo, siendo la identidad absoluta un mero marcar, vacío, sin contenido ni forma, puede contener algún tipo de actividad que sea trascendida? $\mathrm{O}$, lo que es lo mismo: ¿Cómo puede ser activa si la actividad parece encerrar algún tipo de temporalidad? El problema de estas preguntas surge porque el sentido común asocia en todos los casos temporalidad con sucesión o con número en movimiento respecto a lo anterior o lo posterior, teniendo como consecuencia la creencia de que todos los tipos de progresión son temporales siendo, al fin de cuentas, una y la misma cosa (sucesión y temporalidad), cuando en realidad se trata de cosas diferentes. Dejando de lado tal prejuicio y volviendo al tema que nos ocupa, la identidad simple puede verse como una totalidad, unidad cerrada, pero también vacía, que ya es presentación del mero marcar que se halla contenido en sí misma (de ahí que sea también totalidad, "todo-uno"). Por lo tanto, encierra su propia actividad. Recordemos que la identidad absoluta era pura manifestación que se encontraba confinada al manifestarse como actividad (en tanto posibilidad) con lo que se volvía manifestación de manifestación, y así hasta el infinito. Ahora la identidad simple es la manifestación puesta, es decir, la presentación de esa manifestación, (auto)presentación. "A", tal como hemos llamado a la identidad simple, está "puesta" activándose. En efecto, "actividad" no quiere decir puro devenir, como si fuera un intercambio entre el ser y la nada; implica, por el contrario, un "algo" que está en actividad, que se activa: la posibilidad de la manifestación de la manifestación, etc., que se "pone" como presentación.

Es así como lo "puesto" entraña un principio de forma que antes, en la identidad absoluta, no estaba. Pero todavía esta " $\mathrm{A}$ ", al igual que la identidad absoluta, no cuenta con modos determinantes, ${ }^{11}$ sino que permanecen siendo mera marca, aunque con la importante diferencia de forma que proporciona lo ya "puesto". Esta "posición" no debe entenderse como una especie de mano que va y pone, sino como "auto posición" que es, en definitiva, (auto) presentación.

${ }^{11}$ Como, por ejemplo: A $(a ; b ; c \ldots n)$. 


\section{Segunda forma de la identidad}

La identidad simple (" $\mathrm{A}$ "), encierra a la identidad identitaria ("A $\left.=\mathrm{A}^{\prime \prime}\right)$, del mismo modo que la posibilidad de la identidad absoluta hace efectivo el "poner" como (auto)presentación de la identidad simple. En el caso de la identidad identitaria, lo "puesto" es una dualidad de la unicidad, de lo "puesto" como identidad simple. Pues, se trata ahora de la presentación que encierra posibilidad, (auto)presentación de (auto)presentación como el doble de lo "puesto". " $\mathrm{A}$ " se encuentra, en este caso, mediada por sí misma, y esta mediación se hace patente en su propio despliegue como reflejo. En este sentido, apuntamos que hay un alejamiento de sí, ya que se produce un abandono definitivo de lo absoluto (en tanto pasa a considerarse como subsistente). Sin embargo, este reflejo de sí aún continúa siendo una identidad abstracta, sin determinación alguna e incondicionada en contenido, puesto que ahí donde no hay un "otro" tampoco hay una condición. Aunque tenemos un vestigio de condición formal, un primer límite que se produce cuando la identidad consigue reflejarse en sí misma y por el que nos hallamos ante una primera relación.

Si cuando expusimos la identidad simple teníamos una (auto)presentación, ahora dado que la relación de la identidad identitaria es la presentación de la presentación, lo "puesto" de lo "puesto", lo que tenemos es una duplicidad "presentante" que convenimos en llamar "re-presentación", atribuyéndole el sentido de la palabra latina "repraesentatio", como hecho de hacer volver lo presentado. No obstante, la estructura representativa de la identidad identitaria establece una limitación por sí misma. En "A = A" podemos observar una grafía simétrica dada la coincidencia entre función y argumento, forma y contenido. En realidad, habría que escribir "AA" en lugar de "A $=\mathrm{A}^{\prime}$, ya que el " $=$ " presupone un ejercicio de comparación, acto que hemos tratado de combatir para este modelo ontológico formal en $§ 1$. Con todo, por mor de la claridad, dejaremos el signo " $=$ " advirtiendo que no conlleva ningún tipo de tipificación psicológica ni nada por el estilo. Salvaguardando el signo " $="$ ", podemos expresar hasta ahora: una identidad absoluta; una identidad simple ("A") y una identidad identitaria ("A = A").

Recién en " $\mathrm{A}=\mathrm{A}$ " se establece un orden de tiempo, algo que en las otras dos identidades estaba ausente. Tal orden de tiempo es la simultaneidad: "A" es simultáneo a sí mismo. Este "A" abstracto, meramente formal, ahora se encuentra incluido en un orden temporal, pues la simultaneidad implica la no-simultaneidad. Si bien en la simultaneidad el orden es indistinto, este orden comporta una relación. La reflexión, entendida hegelianamente como reflejo de sí, nace de la dualidad que soporta la síntesis entre la identidad absoluta y la identidad simple, es decir, la convergencia de una manifestación de manifestación como un marcar y la mera marca vacía, "lo puesto", 
la presentación. En la presentación de sí, la representación es el "todo-uno" complejo. Al punto que, " $\mathrm{A}=\mathrm{A}$ " es doblamiento de " $\mathrm{A}$ ", imagen de " $\mathrm{A}$ ": mímesis absoluta. Por lo tanto, el orden implícito en la identidad identitaria no es unilateral sino bilateral. Tal es así que si acentuáramos el orden, poniendo en segundo plano la identidad en sí misma obtendríamos especulativamente "A $\leftrightarrow \mathrm{A}$ ". Dejando esto último de lado, la representación de la identidad identitaria, representación como reflejo, mímesis absoluta, significa también un "estar en lugar de...", que por lo que se refiere al sistema bien conocido de la representación, es un "poner" otra vez presente, de donde se toma la imagen como restitución de lo (auto) presentado. Con la representación por medio, la mera marca, duplicada en su presencia, se transforma en signo o símbolo. En "A = A", "A" es signo o símbolo de " $\mathrm{A}$ ", ya que aquello que le atribuíamos a la identidad simple ahora se encuentra en la identidad identitaria de manera reflejada: el signo como reflejo identitario.

\section{Tercera forma de identidad}

Hemos dicho que en la identidad identitaria "A" era reflejo de sí mismo, su propia reflexión. Esto estaba asentado en los dos sentidos en los que se basa la palabra latina "repraesentatio": (1) Como hacer volver lo (auto) presentado, en tanto dualidad simétrica, por la identidad simple y (2) como un "estar en lugar de..." como duplicación de la mera marca, convirtiendo " $\mathrm{A}$ " en signo de " $\mathrm{A}$ ".

Desde el punto de vista de una teoría del reflejo, podemos dividir esta dualidad autorreferencial en aquello que refleja y aquello que es reflejado, aunque lo que es reflejado también refleje, y lo que refleje sea reflejado, recordando que el orden temporal que impera aquí es el de la simultaneidad. Sin embargo, en dicho orden, en el cual obteníamos un primer género de relación, "A" deja de ser totalidad para convertirse en una dualidad relativa. Esta relatividad de "A" consigo misma se deja ver en la distinción del origen con su reflejo, esto quiere decir ni más ni menos que la identidad identitaria conlleva la negación formal de la identidad simple, negación que nosotros escribiremos utilizando, como recurso a favor de ser bien entendido, la partícula lógica " $\neg$ ". De tal manera que, dialécticamente, "A = A" conlleva en origen " $\neg A^{\prime}$. Dejando de lado los argumentos estrictamente lógicos, como el de las "nefastas" consecuencias de la violación del principio de no contradicción, debemos centrarnos estrictamente en el argumento ontológico formal que hace posible la contraposición de " $\mathrm{A}$ " consigo misma. En esta relación identitaria "A" puede concebirse como "lo otro" de sí, la posibilidad pura como (auto) presentación dual de la mera marca, ahora "lo otro", aliquid, su dife- 
rencia. En este sentido, no es del todo correcto escribir "“" $\mathrm{A}$ " $=$ " $\neg \mathrm{A}$ ", si somos del todo precisos lo correcto es escribir: $(" \mathrm{~A}$ " = "A" $)=\neg\left(\right.$ " $\mathrm{A}^{\prime \prime}=$ " $\mathrm{A}$ ") .

Es evidente que entramos en el problema de la negación ontológica, ya que la relación identitaria entraña su propia negación. Pero esta negación debe entenderse formalmente como complemento de una totalidad, esto es, "B". Con la cual, se rompe la simultaneidad y surge la diferencia, la identidad de "lo otro" como "lo puesto": "A" se vuelve signo de otro, se torna referencia.

Al volverse signo de otro ("B"), la actividad de la manifestación deja de ser simultánea para pasar a ser continua, y al ser continua podemos cortar, partir, distanciando partes o eventos. Esto es así porque un todo complejo, como sería " $\mathrm{A}$ " = "B", necesariamente consta de partes, aunque se presenten continuadas, dado que se trata de lo idéntico en su diferencia: lo distinto es lo mismo e igualmente lo indistinto es la distinción de " $\mathrm{A}$ " por un lado y " $\mathrm{B}$ " por otro. Pero aún de este modo, la diferencia no deja de ser formal, cuyo contenido parece estar limitado a pesar de mantenerse vacío. Hay una distinción entre primer y segundo miembro de la identidad, pero ambos son reflejos autorreferenciales de " $\mathrm{A}$ ".

Ahora bien, "A" = "B" lleva implícito el concepto, es decir " $\neg \mathrm{A}$ ", donde el contenido de "A" es su negación, desafiando el principio de no contradicción, como hemos advertido hace algunos momentos. Pero este principio no es original (o al menos no debe suponerse como original) sino que surge cuando se presupone que " $\mathrm{A}$ " es necesariamente incompatible con su complemento; dicho de otra manera, cuando se presupone que el complemento y la posición son antagónicamente diferentes omitiendo la unión que existe entre ambos. El omitir la unión quiere decir hacer surgir el límite, una limitación de "A", lo que es determinado formalmente como " $\mathrm{A}$ " y la negación como otro " $\neg \mathrm{A}$ ". El fundamento de esta negación ya se encuentra en la identidad identitaria, por eso no decimos que es un doblamiento sino un desdoblamiento, ya que el doblamiento, imagen, mímesis absoluta, reflejo, implica un reproducirse, "lo puesto", así mismo, mientras que en la identidad de " $\mathrm{A}$ " = " $\mathrm{B}$ " lo que hay es un producirse como otro, un desdoblamiento encadenante. $Y$ esto es razón por lo cual " $\mathrm{B}$ " puede concebirse como vínculo: ("A" = " $\mathrm{B}$ ") = (" $\mathrm{B}$ " = " $\mathrm{A}$ ").

Surge ahora una peligrosa idea con la que daremos por concluida la parte estrictamente expositiva de este estudio. La idea es que tanto " $\mathrm{B}$ " como los posibles "C"; “ $\mathrm{D}$ "; " $\mathrm{E}$ ". . . etc., pueden concebirse todos ellos como modos de " $\mathrm{A}$ ". Y en cierta medida esto es cierto, pero con un matiz: y es que el término "modo" debe significar "despliegue". Si la identidad simple es "lo puesto" de lo absoluto, entonces cualquier despliegue puede tomarse como modus de la identidad simple. Por lo tanto, si los modos son despliegues de la identidad simple como sus determinaciones, entonces la identidad absoluta debe ser el fundamento, origen, de tales 
determinaciones, y gracias a ella podemos decir que la distinción de las determinaciones identitarias se hace mayor cuanto más partes distinguimos o mayor es la multiplicidad dentro de lo uno-todo, es decir, cuanto mayor sean sus definiciones. En consecuencia, no se debe caer en el error de pensar que la identidad absoluta desaparece en sus derivaciones. La identidad absoluta subsiste siempre-ya presente como actividad, mero marcar vacío, en todas las determinaciones.

\section{Panteísmo identitario y conclusión}

Hemos comenzado este pequeño estudio con dos preguntas rectoras, las cuales hemos desarrollado hasta demarcar los principales tipos de identidad para una ontología formal. Una de las principales conclusiones que podemos extraer de este desarrollo es que en todas las determinaciones subyace un marcar vacío, que viene dado por la subsistencia de la identidad absoluta: la inmanencia de las determinaciones en la identidad absoluta. Sin embargo, no por eso debemos asumir que todas las cosas son modificaciones de la identidad absoluta. Las cosas, en tanto que pueden concebirse como un $x$ indeterminado, son derivadas, pero no modificaciones de la identidad absoluta. La diferencia entre "modificación" y "derivación" es importante porque, mientras que la modificación connota un compuesto de esencia y atributos o en última instancia algo que se modifica, cambia, un cierto contenido, la derivación connota un devenir, un "nacer de...". Queda claro que, si a lo largo de este estudio hemos venido aseverando que la identidad absoluta es un marcar vacío, ausente, sin contenido alguno, entonces no podemos afirmar a la vez que se "modifica", incluso diríamos que la "modificación" de este mero marcar es asimismo mero marcar de mero marcar... etc.

Guiándonos por lo dicho, la identidad absoluta es tal que subsiste pese a sus derivaciones formales, no como un agregado a la cosa o las relaciones que derivan de ella, sino en el devenir mismo de estas relaciones, por lo que es preciso recordar que la subsistencia no es un modo de existencia. La existencia, al caracterizarse por lo "existente", su modus, es principalmente finitud, lo determinado por excelencia y por lo tanto es la diferencia, diferencia relativa. Pero esta diferencia relativa es solamente apariencia relativa en el sentido de un "aparecer como..." relativo. En consecuencia, lo que aparece relativamente siempre está mediado, es mediación de mediación... etc., y por lo tanto negación de la libertad absoluta, porque todo lo constituyente por medio de una mediación está preso de un límite que es su determinación como tal y tal, con lo cual no puede ser absolutamente libre. De tal manera, podemos afirmar que la identidad derivada, identidad relativa, al volverse explícita tematizándose, guarda la verdad de la identidad absoluta; su subsistencia. 
En otras palabras, la verdad es esencialmente identitaria, y viene dada por la subsistencia de la identidad absoluta a través de las identidades derivadas. Por decirlo en forma de máxima: la verdad es la subsistencia de la identidad absoluta en las determinaciones de la facticidad. A esta tesis la llamaremos, por ponerle una etiqueta, "panteísmo identitario". Pero ¿qué quiere decir que la verdad sea identitaria? ¿Acaso no le estamos otorgando cierto privilegio al aspecto positivo de la verdad sin reparar, con igual derecho en su aspecto negativo, esto es, la diferencia? La respuesta a esta última pregunta no admite un sí o un no, sino que merece una explicación de por qué esta pregunta es posible. Suele decirse que en los modos de aparición, ser tal y tal, nace la diferencia mientras que la identidad es algo previo a estos modos, presuponiendo lo que para nosotros es un equívoco, a saber: la dogmática creencia de que, en origen, la diferencia es antagónica a la identidad, sin reparar que cuando decimos "identidad absoluta" también decimos "diferencia absoluta". Si separamos la identidad absoluta de la reflexión, la diferencia que surge a partir de la contraposición reflexiva debe ser una diferencia derivada de "lo puesto", y no una diferencia absoluta. La diferencia absoluta es un mero marcar de marcar... etc. La repetición del mero marcar vacío de contenido es un constante diferir, en esa diferencia está la diferencia, en esa identidad está la identidad. Y en consecuencia la pregunta es inapropiada ya que confunde lo absoluto con lo derivado. Por lo tanto, el panteísmo identitario es también un panteísmo de la diferencia.

Si hacemos hincapié en un aspecto más humano, el panteísmo identitario no significa que todo da igual, una especie de pesimismo grosero, sino que la rueda de la identidad es inmanente a cualquier determinación, subsiste a cualquier diferencia relativa, a cualquier definición. Pero entonces, si afirmamos semejante panteísmo, ¿qué es lo que nos mueve, qué es lo que produce una acción en nosotros, por qué necesitamos hablar de hechos, de verdad, y cómo es que hay cosas que bajo ninguna circunstancia nos parecen idénticas? El mero marcar de la identidad absoluta, en tanto subsistente, yace en el "hablar por hablar". En este "hablar por hablar" la respuesta está en lo que podríamos llamar " $f e$ ": un acto de fe en los enlaces últimos de una realidad indeterminada e inaprensible en sí misma, es lo que hace que este "hablar por hablar" parezca un hablar de algo, es decir, lleno de contenido. Así, está fe se transforma en fe de la fe de la fe... etc., o confianza de la confianza... etc., por lo que la fe en los enlaces no es algo diferente a los propios enlaces: esta fe es ella misma un "enlazar", un enlazar consigo misma. La reflexión en una ontología formal debe llenar el vacío que hay entre el panteísmo identitario y este último acto de fe, teniendo presente y no perdiendo de vista este "hablar por hablar" o "marcar por marcar". 